\title{
Nutrición en el paciente pediátrico en cuidados paliativos
}

\author{
Nutrition in the pediatric patient in palliative care \\ Nutrição no paciente pediátrico em cuidados paliativos
}

Renato Augusto Zorzo ${ }^{1-3^{*}}$, Fabíola de Arruda Leite ${ }^{1,4,5}$, Juliana Cardeal da Costa Zorzo ${ }^{6}$

Recibido: 14 de octubre de 2020. Aceptado para publicación: 1 de diciembre de 2020. https://doi.org/10.35454/rncm.v4n2.218

\section{Resumen}

Los cuidados paliativos son una forma de enfoque que busca la humanización y la comodidad del paciente en una situación de enfermedad crónica, independientemente de la enfermedad subyacente. En esta perspectiva, la búsqueda de la participación conjunta del equipo de atención multidisciplinar, el paciente y su familia es el principal objetivo. La nutrición en el paciente pediátrico en esta situación debe verse como algo que puede ofrecer beneficios, pero también riesgos, y su indicación debe ser objeto de una evaluación minuciosa y continua. Las indicaciones de hidratación y nutrición artificial (HNA) se basan en criterios de autonomía, beneficencia, no maleficencia y justicia. La nutrición en pacientes paliativos debe verse de la misma forma que otros frentes asistenciales, como la antibioticoterapia o la ventilación mecánica. Por tanto, la HNA debe indicarse como una fuente de comodidad para el paciente y la capacidad de mejorar los resultados clínicos, pero cuando se traduce en sufrimiento o malestar mayor que los beneficios, debe contraindicarse o suspenderse.

Palabras clave: cuidados paliativos, pediatría, nutrición artificial.

\section{Summary}

Palliative care is a type of approach that seeks the humanization and wellbeing of patients with chronic conditions, regardless of the underlying disease. In this sense, seeking the joint participation of a multidisciplinary care team, the patient, and his or her family is the main goal. In pediatric patients in this situation, nutrition should be regarded as an approach that is able to provide benefits but that can also bear risks. Therefore, its indication should be subject to a careful and continuous evaluation. Indication for hydration and artificial nutrition (HAN) is based on respect for autonomy, beneficence, non-maleficence, and justice. Nutrition in palliative patients should be addressed in the same ways as other care processes, such as antibiotic therapy or mechanical ventilation. Therefore, HAN should be indicated to improve patient comfort and clinical outcomes, but when HAN results in suffering or discomfort, outweighing its benefits it should be contraindicated or discontinued.

Keywords: Palliative Care; Pediatrics; Artificial Nutrition.

\section{Resumo}

Os cuidados paliativos são uma forma de abordagem que busca a humanização e o conforto do paciente em situação de doença crônica, independente da doença de base. Nesta perspectiva, o objetivo principal é a busca da participação conjunta da equipe multidisciplinar, do paciente e de sua família. A nutrição do paciente pediátrico em esta situação deve ser vista como algo que pode trazer benefícios, assim como riscos, e sua indicação deve ser objeto de avaliação criteriosa e contínua. As indicações de hidratação e nutrição artificial (HNA) são baseadas em critérios de autonomia, benefício ao paciente, e equidadade. A nutrição em pacientes paliativos deve ser vista da mesma forma que outras frentes de cuidado assistencial, como a antibioticoterapia ou a ventilação mecânica.

Portanto, a HNA deve ser indicada como fonte de conforto para o paciente e a capacidade de melhorar os desfechos clínicos, mas quando o sofrimento ou desconforto è maior do que os benefícios, deve ser contra-indicada ou suspensa.

Palavras-chave: cuidados paliativos, pediatria, nutrição artificial.

\footnotetext{
Facultad de Medicina de Ribeirão Preto, Universidad de São Paulo, Brasil.

2 Equipo Multidisciplinario de Terapia Nutricional, Hospital Estadual de Américo Brasiliense, complejo Hospital das Clínicas de la Facultad de Medicina de Ribeirão Preto, Universidad de São Paulo, Brasil.

*Correspondencia: Renato Augusto Zorzo. dr.zorzo@gmail.com
}

\footnotetext{
3 Facultad de Medicina, Centro Universitario Estácio de Ribeirão Preto, Brasil.

4 Hospital da Criança, São Paulo, Brasil.

5 Facultad de Medicina, Centro Universitario Barão de Mauá, Ribeirão Preto, Brasil.

6 Unidad de Pediatría Funcional de la Unidad de Urgencias, Hospital das Clínicas de la Facultad de Medicina de Ribeirão Preto, Universidad de São Paulo, Brasil.
} 


\section{INTRODUCCIÓN}

Los cuidados paliativos se pueden ofrecer a pacientes de todos los grupos de edad con enfermedades graves y limitantes (para las que no hay esperanza de cura y la muerte puede ser temprana) o potencialmente mortales (aquellos para quienes existe un tratamiento curativo, pero este puede fallar), ya sea aguda o crónica. En pediatría nos ocupamos de la diversidad de enfermedades de base, ya que el rango de edad pediátrico varía desde la atención en los primeros días de vida (neonatología) hasta la fase de transición entre la adolescencia y la edad adulta ${ }^{(1)}$.

De acuerdo con las recomendaciones internacionales, este abordaje debe iniciarse desde el diagnóstico y mantenerse a lo largo de la evolución, en sus diferentes fases, e intensificarse al final de la vida (evolución de la enfermedad). No se trata solo de garantizar una muerte digna al niño, sino también de asegurar su calidad de vida, aliviar sus síntomas y ofrecer apoyo a los familiares durante todo el proceso de la enfermedad y en la fase de duelo ${ }^{(2-4)}$.

Para atender las demandas de estos pacientes y sus familias, el trabajo en equipo interdisciplinario es fundamental, y debe coordinarse para tener claros los objetivos asistenciales (manejo de síntomas, rehabilitación y calidad de vida), las limitaciones que ocasiona la enfermedad, la planificación futura y los valores involucrados en la elaboración del plan de cuidados avanzados (que guiará los límites de inversión terapéutica considerados proporcionales). Esta planificación será individualizada y flexibilizada, y se debe revisar de acuerdo con la evolución de la enfermedad y ajustarse a las necesidades del niño y su familia, con el foco principal en la atención integral y el alivio del sufrimiento ${ }^{(1,5)}$.

Con los avances tecnológicos, la gama de tratamientos para los niños con afecciones limitantes o potencialmente mortales es amplia y, en muchos casos, el uso de estos recursos implica un aumento de la esperanza de vida. Sin embargo, debemos evaluar la carga que esta oferta de extensión de tiempo traerá sobre el niño y su familia. A menudo, el apoyo propuesto tendrá una carga de sufrimiento y dolor que hará imposible que el niño tenga algún beneficio con el mayor tiempo de supervivencia ${ }^{(1,6,7)}$.

El final de la vida es un momento de gran sufrimiento para los padres y de una intensa carga emocional, que requerirá un apoyo adecuado, con profesionales técnicamente capacitados en el manejo de síntomas físicos, psicológicos, sociales y espirituales. La literatura apunta a una diversidad de síntomas físicos en la fase final de la vida, como dolor, disnea, fatiga, convulsiones, disfagia y síntomas gastrointestinales, que impactan negativamente tanto en el niño como en sus familiares, lo cual puede contribuir a un complicado proceso de duelo ${ }^{(1,8)}$.

$\mathrm{Al}$ evaluar las cuestiones relacionadas con la pediatría en el contexto de los cuidados paliativos, es posible identificar que los valores socioculturales y religiosos tienen una gran influencia en la toma de decisiones. Los profesionales de la salud deben respetar estos valores y cuidar el interés superior del niño, considerando los principios bioéticos en este proceso de atención (autonomía, beneficencia, no maleficencia y justicia) ${ }^{(1,7)}$. Sin embargo, incluso respetando los valores familiares y el principio de autonomía, en ocasiones no se evitarán desajustes de objetivos y conflictos que interferirán en el momento de la toma de decisiones. Estos suelen ocurrir por interpretación divergente de valores y proporcionalidad terapéutica, lo que genera incertidumbre sobre cuál es la mejor opción: iniciar, mantener o suspender algún tratamiento o soporte vital artificial ${ }^{(6,7,9)}$.

En pediatría son frecuentes las divergencias, ya que una de las formas de demostrar afecto, amor y cuidado es a través de la comida. Existe una relación simbólica en la realización de este acto de cuidado básico de la vida por parte de los padres. Cuando los familiares se ven privados de la alimentación oral a sus hijos, y se requiere el uso de tecnología y dispositivos, como sondas o gastrostomías, estos pueden experimentar sentimientos como miedo, frustración, ansiedad y culpa, y el acto de administrar hidratación y nutrición artificial (HNA) convertirse en una fuente de estrés y, a veces, aumentar el gasto $^{(10)}$.

El objetivo de esta revisión es presentar los aspectos más relevantes de la nutrición en el paciente pediátrico en cuidados paliativos.

\section{INDICACIONES DE LA HIDRATACIÓN Y NUTRICIÓN ARTIFICIAL}

Algunas enfermedades avanzarán con una progresión de dificultad para succionar o masticar y tragar, como enfermedades neurológicas, errores innatos del metabolismo o tumores del sistema nervioso central. La pérdida de esta capacidad de alimentación genera ansiedad en la familia, ya que representa el agravamiento clínico y la evolución de la enfermedad. En este momento, el enfoque compasivo hacia la familia ayudará a aceptar la alimentación artificial asistida o la medicación. Destacamos que en esta transición de la vía oral al uso de dispositivos, la HNA éticamente se convierte en un procedimiento médico ${ }^{(11)}$. 
¿Cómo valorar si existe o no indicación de introducción de hidratación y dieta en un momento determinado o condición clínica? ¿Cómo preparar y ayudar a la familia en la toma de decisiones? ¿Cuándo la suspensión del soporte nutricional puede traer beneficios o prevenir daños al paciente? ¿Existe un protocolo eficaz para ayudar a los profesionales de la salud en esta decisión de mantener o suspender la HNA ${ }^{(11-15)}$. Estas preguntas se pueden responder con la ayuda de profesionales en cuidados paliativos pediátricos, nutriología, logopedia y nutrición, ya que las vías alternativas también serán de utilidad en la administración de medicamentos, no solo para la HNA. En las condiciones donde está indicada la gastrostomía, el equipo de atención y el quirúrgico deben discutir las mejores opciones y compartir con los familiares, acogiendo sus dudas y ansiedades, y exponiendo los riesgos y beneficios del procedimiento ${ }^{(16)}$.

Algunas enfermedades requerirán soporte de HNA intravenoso, es decir, nutrición parenteral, como en los casos de síndrome del intestino corto, cambios en la absorción intestinal, dismotilidad gastrointestinal, mucositis severa por quimioterapia, desnutrición severa, enfermedad hepática, trasplantes sólidos o de médula ósea, prematuridad, displasia broncopulmonar grave y otras enfermedades agudas potencialmente mortales (politraumatismos, infecciones y quemaduras graves, entre otras) ${ }^{(17)}$.

Si existen dudas sobre la introducción de vías alternativas a la HNA en cuidados paliativos, los conflictos pueden aumentar al discutir el mantenimiento o suspensión de la HNA por vía parenteral, cuando el equipo médico cree que mantener estos cuidados puede ser una forma de prolongar el sufrimiento, especialmente cuando se trata de una enfermedad neonatal mortal o en fase terminal ${ }^{(18)}$.

Son escasos los estudios que discuten la nutrición parenteral en el contexto de los cuidados paliativos pediátricos. En algunas patologías, esta será la única vía posible a la HNA, como en situaciones de intestino corto. En casos de fallo de acceso venoso e imposibilidad de HNA enteral, estos niños tendrán un desenlace desfavorable. En estos pacientes, la vía subcutánea puede ser una opción de hidratación, pero no brindará el soporte nutricional necesario para el mantenimiento basal por un período prolongado ${ }^{(19)}$.

\section{CONSIDERACIONES SOBRE LA TERAPIA NUTRICIONAL PARA EL PACIENTE PEDIÁTRICO}

Todas las personas necesitan comida e hidratación para sobrevivir. Cuando esta necesidad se satisface de forma espontánea, no hay indicación de intervención; sin embargo, cuando un paciente no puede ingerir lo suficiente se establece un problema. La solución racional es considerar la intervención de $\mathrm{HNA}^{(20)}$.

$\mathrm{Al}$ diseñar el plan terapéutico para el paciente pediátrico en cuidados paliativos se debe considerar que la HNA no está libre de riesgos. Todo lo contrario, se trata de intervenciones médicas instituidas tras la valoración individual y la toma de decisiones del paciente ${ }^{(18)}$. Existen muchas preguntas sobre los beneficios de mantener la HNA en la fase terminal debido al riesgo de desencadenar o empeorar los síntomas ${ }^{(6-8)}$.

La literatura indica que la deshidratación puede desencadenar síntomas desagradables, como fatiga, náuseas, fiebre, sensación de sed y boca seca, deterioro cognitivo, y convulsiones, secundarias a hipernatremia ${ }^{(6,13,18)}$. Por otro lado, la hidratación artificial al final de la vida puede intensificar los síntomas y agravar el sufrimiento ${ }^{(18,21)}$.

La decisión de iniciar la HNA debe tener en cuenta si el procedimiento proporcionará mayores beneficios que riesgos. Si los riesgos superan a los beneficios, el equipo médico tiene la obligación de no indicarlo. Los niños en cuidados paliativos a menudo se encuentran en una situación pasiva de dolor, reciben medicamentos que alteran el gusto o son anorexígenos, y tienen riesgo de depresión y trastornos gastrointestinales ${ }^{(18,20,21)}$.

En los niños que pueden recibir dieta o hidratación oral se debe tener cuidado del riesgo de broncoaspiración, y se recomienda una evaluación del habla y el lenguaje si se sospecha de algún grado de trastorno de la deglución. En este escenario, la indicación de la terapia nutricional es capaz de reducir el riesgo de asfixia, aspiración, vómitos y disnea. Desde la perspectiva de la familia, el inicio de la HNA brinda una sensación de seguridad, en el sentido de garantizar que la desnutrición no acelerará el proceso de muerte y morir $^{(18,20,21)}$.

La decisión de iniciar la HNA debe ser individualizada $y$, una vez indicada al paciente con cuidados proporcionales, esta debe revisarse con frecuencia, ya que cuando el procedimiento ocasiona una situación de malestar y sufrimiento constante, la discusión de si continuar o interrumpir cobra relevancia. Según la progresión de la enfermedad, si la HNA comienza a ofrecer más sufrimiento que beneficios, esta debe detenerse. La decisión que se debe tomar siempre debe involucrar no solo al equipo de atención, sino a la familia del paciente. Si se toma la decisión de suspender la HNA se deben mantener todos los demás frentes de cuidados paliativos ${ }^{(18,20,22)}$.

La HNA se puede realizar mediante sonda nasogástrica o nasoyeyunal, gastrostomía, intravenosa o hipo- 
dermoclisis. La elección de la vía sigue la secuencia tradicional, es decir, la vía oral preferencial, siempre que sea posible, vía enteral, como segunda opción, seguida de intravenosa o hipodermoclisis (Figura 1). Siempre se debe preferir el uso de la vía digestiva, ya que las vías parenterales son más invasivas. Esto siempre se debe discutir con gran discreción, ya que agrega malestar y dificultades en el manejo práctico para la familia ${ }^{(18,20)}$.

A medida que avanza la enfermedad subyacente y el niño pierde su autonomía, los riesgos de la HNA comienzan a aumentar, hasta el punto de que los beneficios no se compensan. Llega un momento en que la prescripción nutricional empieza a tener un objetivo más de ofrecer el placer de la percepción de los gustos, que el de nutrir adecuadamente. En las etapas más avanzadas de la enfermedad, cuando el proceso de morir está avanzado, es común que el niño ni siquiera pueda alimentarse por vía oral. En esta situación, la provisión de alimentos y líquidos solo servirá para brindar alivio y placer ${ }^{(18)}$. Sin embargo, se debe considerar que estas etapas no tienen una definición clara, por lo que el mejor momento para decidir sobre el inicio, modificación e interrupción de la HNA no siempre es una ejecución fácil por parte del equipo médico ${ }^{(18)}$.

En situaciones de final de vida en las que el equipo considera necesario mantener la HNA, ya sea por la condición de minimizar los síntomas o por solicitud de la familia, se recomienda un suministro de agua reducido, de alrededor $25 \%$ al $50 \%$ de las necesidades basales.

\section{¿CUÁNDO INDICAR LA TERAPIA NUTRICIONAL?}

La indicación clásica de terapia nutricional comienza cuando el niño no puede alcanzar el $60 \%-80 \%$ del valor energético total (VET) durante 10 días consecutivos $^{(22)}$. Sin embargo, se deben tener en cuenta otros factores en el paciente en cuidados paliativos ${ }^{(20,21,23)}$. Para que la terapia nutricional se indique debe existir un consenso entre el equipo de cuidados paliativos y la familia sobre los riesgos, beneficios y objetivos de la intervención. Siempre que sea posible, el niño también debe participar en la decisión. Esta debe involucrar mínimamente los siguientes factores ${ }^{(20,21)}$ :

- Potencial para mejorar la caquexia y la anorexia.

- Consideración de posibles efectos adversos, como aspiración, estreñimiento y diarrea.

- Consideración de que la intervención debe ser cómoda para el paciente mientras se aplica.

- Consideración sobre el sufrimiento que puede acarrear al paciente, en todos los ámbitos (físico, psicológico, social y espiritual).

- Fortalecimiento de las relaciones entre el paciente, su familia y el equipo de atención

- Manejo de los síntomas de la enfermedad principal.

- Observación de los principios éticos de autonomía, beneficencia, no maleficencia y justicia.

Siempre se debe tener en cuenta la autonomía del paciente. En este escenario, esta debe entenderse como el respeto a la decisión del paciente y sus representantes legales en relación con la denegación del tratamiento propuesto, aun cuando exista una indicación médica de intervención, y el resultado negativo en acelerar el proceso de muerte y agonizar ${ }^{(20,24)}$.

Los principios de beneficencia y no maleficencia también deben impregnar el proceso de toma de decisiones. En los casos en los que no se tenga certeza de que la intervención sea beneficiosa se debe iniciar la HNA a modo de prueba, y posponer la decisión final a

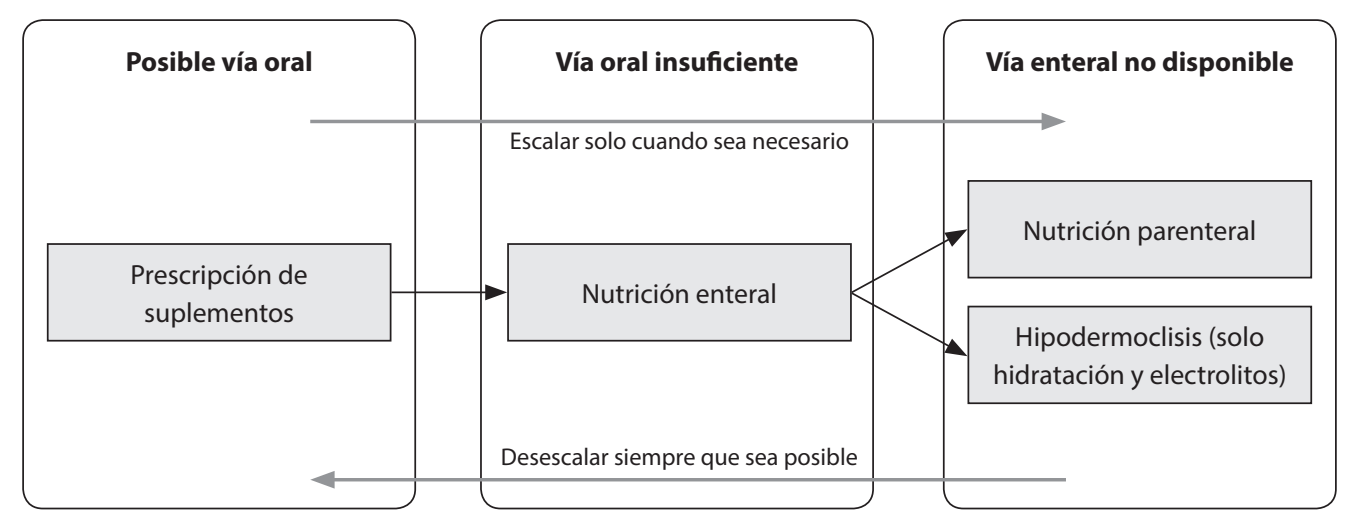

Figura 1. Diagrama de flujo para la elección de la vía de administración de la hidratación y de la nutrición artificial(20). 
un segundo momento ${ }^{(20)}$. En el caso de decidir no iniciar la HNA, o interrumpirla, este acto no debe verse como una prohibición de alimentar al paciente; por el contrario, se debe tener en cuenta el concepto de alimentación confortable, ya que el principio es siempre ofrecer alivio y comodidad ${ }^{(20,21)}$.

\section{PRESCRIPCIÓN DE LA HNA}

La HNA debe considerarse cuando el paciente no puede alcanzar los objetivos del VET prescritos. Inicialmente, la prescripción de suplementos orales y modulares tiene la función de mejorar el aporte nutricional oral de estos pacientes ${ }^{(20,24)}$.

Desde el momento en que la vía oral se vuelve insuficiente, incluso con el uso de suplementos, se puede considerar la vía enteral. De manera similar, tan pronto como el tracto digestivo se vuelve imposible o insuficiente, se puede instituir el tracto parenteral ${ }^{(20,21,23)}$. La transición

Tabla 1. Valor energético total por peso corporal, según Holliday \& Segar y según ASPEN ${ }^{(24,25)}$

\begin{tabular}{|c|c|}
\hline \multicolumn{2}{|c|}{ Según Holliday \& Segar, $1957^{(24)}$} \\
\hline Peso & VET diario \\
\hline Hasta 10 kg & $100 \mathrm{kcal} / \mathrm{kg}$ \\
\hline De $11-20$ kg & $\begin{array}{c}1000 \mathrm{kcal}+50 \mathrm{kcal} / \mathrm{kg} \text { por cada } \mathrm{kg} \text { de más } \\
\text { de } 10 \mathrm{~kg}\end{array}$ \\
\hline Más de 20 kg & $\begin{array}{c}1500 \mathrm{kcal}+20 \mathrm{kcal} / \mathrm{kg} \text { por cada kg de más } \\
\text { de } 20 \mathrm{~kg}\end{array}$ \\
\hline \multicolumn{2}{|r|}{ Según ASPEN, 2002(25) } \\
\hline Edad & VET diario \\
\hline De 0-1 año & $90-120 \mathrm{kcal} / \mathrm{kg}$ \\
\hline De 1-7 años & $75-90 \mathrm{kcal} / \mathrm{kg}$ \\
\hline De 7-12 años & $60-75 \mathrm{kcal} / \mathrm{kg}$ \\
\hline De 12-18 años & $30-60 \mathrm{kcal} / \mathrm{kg}$ \\
\hline
\end{tabular}

APSEN: American Society of Parenteral and Enteral Nutrition; VET: valor energético total. entre las vías de administración siempre debe revisarse en función de la dinámica del paciente. Si un paciente en nutrición parenteral vuelve a tener habilitada su vía enteral, se debe realizar la reversión, de la misma forma con el paciente en nutrición enteral que vuelve a tener la vía oral lo más posible. La elección siempre debe ser la de la vía más fisiológica posible $(20,21,23,24)$.

La prescripción de terapia nutricional, cuando está indicada, no tiene por qué alcanzar necesariamente el objetivo habitual del VET y proteínas para el paciente, considerando la proporcionalidad del tratamiento, con un enfoque en una prescripción individualizada ${ }^{(20,21,23,24)}$. Los objetivos de energía y proteínas se muestran en las Tablas 1 y $2^{(24)}$.

\section{CONSIDERACIONES ÉTICAS}

El acto de criar a un ser querido es una forma de expresar cariño, por lo que este peso simbólico puede llevar

Tabla 2. Requerimientos de proteínas por $\operatorname{edad}^{(25,26)}$

\begin{tabular}{|c|c|}
\hline \multicolumn{2}{|c|}{ Según el Institute of Medicine (IOM), $2005^{(26)}$} \\
\hline Edad & $\begin{array}{l}\text { Requerimientos de } \\
\text { proteínas }\end{array}$ \\
\hline De 0-6 meses & 9,1 g/día \\
\hline De 7-12 meses & 11 g/día \\
\hline De 1-3 años & 13 g/día \\
\hline De 4-8 años & 19 g/día \\
\hline De 9-13 años & 34 g/día \\
\hline De 14-18 años & $\begin{array}{c}\text { Mujer: } 46 \text { g/día; hombre: } 52 \\
\text { g/día }\end{array}$ \\
\hline \multicolumn{2}{|c|}{ Según ASPEN, 2002 ${ }^{(25)}$} \\
\hline Tipo de paciente & $\begin{array}{l}\text { Requerimientos de } \\
\text { proteínas }\end{array}$ \\
\hline BPAN & $3,4 \mathrm{~g} / \mathrm{kg} / \mathrm{día}$ \\
\hline RNAT AIG & $2,3 \mathrm{~g} / \mathrm{kg} / \mathrm{día}$ \\
\hline Adolescentes (masculino) & $0,9 \mathrm{~g} / \mathrm{kg} / \mathrm{dí} a$ \\
\hline Adolescentes (femenino) & 0,8 g/kg/día \\
\hline Pacientes críticos pediátricos & 1,5 g/kg/día \\
\hline
\end{tabular}

AIG: adecuado para la edad gestacional; BPAN: bajo peso al nacer; RNAT: recién nacido a término. 
a la familia a expresar el deseo de iniciar la HNA más por motivos emocionales que racionales, aun cuando la falta de beneficios sea evidente ${ }^{(18,21)}$.

La alimentación y la hidratación tienen un aspecto afectivo muy intenso, y se relacionan con celebraciones y momentos de alegría en muchas culturas. El vínculo psicológico y afectivo del acto de alimentarse proviene de los instintos primitivos de supervivencia. Cuestiones como suspender la hidratación y alimentar a un recién nacido o un niño pueden desencadenar una gran incomodidad y ser una fuente de conflictos agotadores ${ }^{(7,18,27,28)}$.

Para los padres, muchas veces aceptar suspender este apoyo puede generar angustia, culpa y aumentar el sufrimiento por la muerte del niño; sin embargo, es fundamental que el equipo de atención tenga como objetivo gestionar el sufrimiento y velar por el mejor interés del niño y, en algunas situaciones, la opción de suspender la HNA será una forma de manejar los síntomas ${ }^{(14,18)}$.

Debido a que la HNA se considera un procedimiento médico, es decir, un soporte vital artificial, es éticamente posible suspenderla en la fase terminal, cuando el paciente no tiene deseos ni capacidad para alimentarse o hidratarse. Así, la HNA no se diferencia de otros recursos de soporte vital artificial ${ }^{(6,7,13,14,18)}$.

Es de destacar que desde un punto de vista ético no existe diferencia entre la decisión de no iniciar o suspender la $\mathrm{HNA}^{(13,18)}$. El equipo debe evaluar el apoyo ofrecido y decidir si el tratamiento realmente se considera sin beneficios o desproporcionado, es decir, cuál es el mejor interés del niño, considerando los riesgos y daños, y los posibles beneficios de la HNA. En algunas situaciones, como muerte cerebral, cáncer avanzado y anencefalia, esta toma de decisiones es más clara ${ }^{(6,13,15)}$.

La recomendación es que la decisión sobre la HNA esté en el plan de atención avanzada, discutido antes del momento de la crisis. De esta forma, los padres tendrán tiempo para comprender la evolución de la enfermedad, las necesidades del niño y la proporcionalidad de los tratamientos. En situaciones en las que exista una divergencia entre los valores del equipo y la familia se recomienda la opinión de otros profesionales, preferiblemente del comité de ética o bioética. Aun así, en situaciones como, por ejemplo, el estado vegetativo persistente, se puede judicializar el tema de mantener o suspender la $\mathrm{HNA}^{(6,18)}$.

\section{CONCLUSIONES}

El abordaje nutricional de los pacientes pediátricos en cuidados paliativos debe ser individualizado, sin pará- metros fijos para decidir cuándo iniciar la HNA. El proceso de toma de decisiones para iniciar la HNA debe involucrar al equipo de cuidado infantil, representantes legales $y$, cuando sea posible, al niño mismo.

La HNA debe prescribirse teniendo en cuenta los principios de autonomía, beneficencia y no maleficencia. Si los beneficios no superan los riesgos no se debe iniciar el procedimiento. Al indicar la HNA, el seguimiento debe ser continuo, ya que la decisión de mantenerla o suspenderla será frecuente.

La ruta debe ser lo más fisiológica posible. La programación de la ruta debe realizarse solo cuando sea necesaria, y siempre que sea posible. La HNA tiene como principal objetivo ofrecer comodidad y dignidad, por lo que en la medida en que el sufrimiento del procedimiento supere los beneficios, esta se debe suspender.

\section{Financiamiento}

El presente estudio no tuvo financiación.

\section{Conflicto de intereses}

Ninguno.

\section{Declaración de autoría}

Todos los autores contribuyeron por igual. Todos los autores aprobaron la versión final del manuscrito.

\section{Referencias bibliográficas}

1. Himelstein BPB, Hilden JMJJM, Boldt AM, Weissman D, Klick JC, Hauer J, et al. Pediatric palliative care. N Engl J Med. 2004;350(17):1752-62. doi: http://dx.doi.org/10.1016/j. cppeds.2010.05.001.

2. American Academy of Pediatrics. Committee on Bioethics and Committee on Hospital Care. Palliative care for children. Pediatrics. 2000;106(2 Pt 1):351-7.

3. Sisk BA, Feudtner C, Bluebond-Langner M, Sourkes B, Hinds PS, Wolfe J. Response to suffering of the seriously ill child: a history of palliative care for children. Pediatrics. 2020;145(1):e20191741. doi: 10.1542/peds.2019-1741.

4. Friedrichsdorf S, Bruera E. Delivering pediatric palliative care: from denial, palliphobia, pallilalia to palliactive. Children. 2018;5(9):120.

5. Jordan M, Keefer PM, Lee Y-LA, Meade K, Snaman JM, Wolfe J, et al. Top ten tips palliative care clinicians should know about caring for children. J Palliat Med. 2018;21(12):1783-9. doi: 10.1089/jpm.2018.0482.

6. Larcher V, Craig F, Bhogal K, Wilkinson D, Brierley J; Royal College of Paediatrics and Child Health. Making decisions to limit treatment in life-limiting and life-threatening condi- 
tions in children: a framework for practice. Arch Dis Child. 2015;100:s3-23. doi: 10.1136/archdischild-2014-306666.

7. Carter BS, Leuthner SR. The ethics of withholding/ withdrawing nutrition in the newborn. Semin Perinatol. 2003;27(6):480-7. doi: 10.1053/j.semperi.2003.10.007.

8. Knops RRG, Kremer LCM, Verhagen AAE. Paediatric palliative care: recommendations for treatment of symptoms in the Netherlands Palliative care in other conditions. BMC Palliat Care. 2015;14(1):1-8. doi: doi.org/10.1186/s12904-0150054-7.

9. Himelstein B. Palliative care reviews palliative care for infants, children, adolescents, and their families. J Palliat Med. 2006;9(1):163-81. doi: 10.1089/jpm.2006.9.163.

10. Edwards S, Davis AM, Bruce A, Mousa H, Lyman B, Cocjin J, et al. Caring for tube-fed children: a review of management, tube weaning, and emotional considerations. J Parenter Enter Nutr. 2016;40(5):616-22. doi: https://doi. org/10.1177/0148607115577449.

11. Mahant S, Cohen E, Nelson KE, Rosenbaum P. Decisionmaking around gastrostomy tube feeding in children with neurologic impairment: Engaging effectively with families. Paediatr Child Heal. 2018;23(3):209-13. doi: 10.1093/pch/ pxx193.

12. Kaufman J, Vichayavilas P, Rannie M, Peyton C, Carpenter E, Hull D, et al. Improved nutrition delivery and nutrition status in critically ill children with heart disease. Pediatrics. 2015;135(3):e717-25. doi: 10.1542/peds.2014-1835.

13. Diekema DS, Botkin JR; Committee on Bioethics. Clinical report--Forgoing medically provided nutrition and hydration in children. Pediatrics. 2009;124(2):813-22. doi: 10.1542/ peds.2009-1299.

14. Rapoport A, Shaheed J, Newman C, Rugg M, Steele R. Parental perceptions of forgoing artificial nutrition and hydration during end-of-life care. Pediatrics. 2013;131(5):861-9.

15. Ganzini L. Artificial nutrition and hydration at the end of life: ethics and evidence. Palliat Support Care. 2006;4(2):135-43. doi: $10.1017 /$ S1478951506060196.

16. Mahant S, Jovcevska V, Cohen E. Decision-making around gastrostomy-feeding in children with neurologic disabilities. Pediatrics. 2011;127(6):e1471-81. doi: 10.1542/peds.20103007.

17. Pedrón Giner C, Cuervas-Mons Vendrell M, Galera Martínez R, Gómez López L, Gomis Muñoz P, Irastorza Terradillos I, et al. Guía de práctica clínica SENPE/SEGHNP/SEFH sobre nutrición parenteral pediátrica. Nutr Hosp. 2017;34(3):74558. doi: 10.20960/nh.1116.

18. Anderson AK, Burke K, Bendle L, Koh M, McCulloch R, Breen M. Artificial nutrition and hydration for children and young people towards end of life: consensus guidelines across four specialist paediatric palliative care centres. BMJ Support Palliat Care. 2019 19:bmjspcare-2019-001909. doi: 10.1136/ bmjspcare-2019-001909.
19. Caccialanza R, Constans T, Cotogni P, Zaloga GP, PontesArruda A. Subcutaneous infusion of fluids for hydration or nutrition: a review. JPEN J Parenter Enteral Nutr. 2018;42(2):296-307. doi: 10.1177/0148607116676593.

20. Druml C, Ballmer PE, Druml W, Oehmichen F, Shenkin A, Singer P, et al. ESPEN guideline on ethical aspects of artificial nutrition and hydration. Clin Nutr. 2016;35(3):545-56. doi: 10.1016/j.clnu.2016.02.006.

21. Nutrição no pacientes em cuidados paliativos. Departamento cientifico de suporte nutricional \& Departamento cientifico de medicina da dor e cuidados paliativos. Sociedade Brasileira de Pediatria [Internet]. 2019. (Consultado el 1 de octubre de 2020). Disponible en: https://www.sbp.com.br/filead$\mathrm{min} /$ user_upload/2017/03/Medicina-da-Dor-CuidadosPaliativos.pdf

22. Braegger C, Decsi T, Dias JA, Hartman C, Kolacek S, Koletzko B, Koletzko S, Mihatsch W, Moreno L, Puntis J, Shamir R, Szajewska H, Turck D, van Goudoever J; ESPGHAN Committee on Nutrition: Practical approach to paediatric enteral nutrition: a comment by the ESPGHAN committee on nutrition. J Pediatr Gastroenterol Nutr. 2010 ;51(1):11022. doi: 10.1097/MPG.0b013e3181d336d2.

23. Cuidados paliativos pediátricos: o que são e qual sua importância? Cuidando da criança em todos os momentos. Departamento cientifico de medicina da dor e cuidados paliativos [Internet]. Sociedade Brasileira de Pediatria. 2019. (Consultado el 1 de octubre de 2020) Disponible en: https://www.sbp.com.br/fileadmin/user_upload/2017/03/ Medicina-da-Dor-Cuidados-Paliativos.pdf

24. Holliday MA, Segar WE. The maintenance need for water in parenteral fluid therapy. Pediatrics. 1957;19(5):823-32.

25. ASPEN Board of Directors and the Clinical Guidelines Task Force. Guidelines for the use of parenteral and enteral nutrition in adult and pediatric patients. JPEN J Parenter Enteral Nutr. 2002;26(1):1SA-138SA.

26. Trumbo P, Schlicker S, Yates AA, Poos M; Food and Nutrition Board of the Institute of Medicine, The National Academies. Dietary reference intakes for energy, carbohydrate, fiber, fat, fatty acids, cholesterol, protein and amino acids. J Am Diet Assoc. 2002;102(11):1621-30. doi: 10.1016/s00028223(02)90346-9.

27. Fournier V, Belghiti E, Brunet L, Spranzi M. Withdrawal of artificial nutrition and hydration in neonatal intensive care: parents' and healthcare practitioners' views. Med Heal Care Philos. 2017;20(3):365-71.

28. Parravicini E, Daho M, Foe G, Steinwurtzel R, Byrne M. Parental assessment of comfort in newborns affected by lifelimiting conditions treated by a standardized neonatal comfort care program. J Perinatol. 2018;38(2):142-7. doi: 10.1038/ jp.2017.160. 